\title{
De la créativité lexicale à l'hybridité générique et vocale: une analyse stylistique de Le-fils-de-la- femme-mâle de Maurice Bandaman
}

\section{From Lexical Creativeness to Generic and Vocal Hybridity: A Stylistic Analysis of Maurice Bandama's Le-fils-de-la- femme-mâle}

\author{
Daouda COULIBALY'
}

${ }^{1}$ Assistant, Université Peleforo Gon Coulibaly, UFR des Lettres et des Arts, Département de Lettres Modernes, Korhogo-Côte d'Ivoire

ORCID: D.C. 0000-0002-1145-2681

\section{Corresponding author: \\ Daouda COULIBALY, \\ Université Peleforo Gon Coulibaly, UFR des Lettres et des Arts, Département de Lettres Modernes, BP 1328, Korhogo, Côte d'Ivoire E-mail: d.coulibaly09@yahoo.com}

Submitted: 15.02 .2020

Accepted: 01.07.2020

Citation: Coulibaly, D. (2020). De la créativité lexicale à l'hybridité générique et vocale: une analyse stylistique de Le-fils-de-la-femme-mâle de Maurice Bandaman. Litera, 30(2), 543-562. https://doi.org/10.26650/LITERA2020-0038

\section{RÉSUMÉ}

La stylistique est une science née des travaux de Charles Bally au début du XX⿳亠丷⿵冂丶 siècle. Elle a pour objet le texte littéraire. Pour le décrypter, la stylistique s'appuie sur des méthodes et des concepts issus des sciences du langage. Elle est donc efficace pour mettre au jour les différentes subtilités du roman africain qui ne cesse de se régénérer à partir des ressources de l'oralité et du patrimoine linguistique africain. Cette régénération se traduit par une africanisation de la langue d'écriture. La relexicalisation est au cœur de la praxis verbale. Elle se matérialise par l'enchâssement d'ethno-textes oraux dans le roman. Cette créativité est une forme de subversion discursive qui se caractérise par une esthétique polyphonique et transgénérique. Le-fils-de-la-femme-mâle de Maurice Bandaman est conforme à cette norme. La configuration typographique montre que les fondements énonciatifs de l'œuvre reposent sur la structure du conte traditionnel africain. De ce fait, le roman s'oppose à la narration traditionnelle. En outre, l'insertion du conte africain dans la structure du roman ne repose sur aucune règle stable. Le discours est fondé sur une pratique intertextuelle qui bouleverse l'architectonique du roman classique. II rompt avec I'horizon d'attente et impose une autre modalité de réception qui procure de la jouissance au lecteur obvie. Ce travail met en évidence l'ensemble des phénomènes énonciatifs, linguistiques et discursifs qui participent à l'authenticité de cette œuvre. Mots-clés: Stylistique, hybridité générique, intertextualité, scène d'énonciation, polyphonie

\section{ABSTRACT}

Stylistics is a science born from the works of Charles Bally at the start of the $20^{\text {th }}$ century. It centers on literary texts. To decipher them, stylistics focuses on methods and concepts from the language sciences. It is therefore effective in bringing to light the various subtleties of the African novel which continues to regenerate from the resources of African language and linguistic heritage. This regeneration results in an Africanization of the writing language. It follows that re-lexicalization is at the heart of verbal praxis. It is materialized by the embedding of oral ethno-texts in the 
novel. This creativity is a form of discursive subversion which is characterized by a polyphonic and transgeneric aesthetics. Le-fils-de-la-femme-mâle by Maurice Bandaman conforms to this standard. The typographical configuration shows that the enunciative foundations of the work rests on the structure of the traditional African tale. As a result, the novel resists the norms of traditional storytelling. Furthermore, the insertion of the African tale into the structure of the novel is not based on any stable rule. The discourse is based on an intertextual practice that upsets the architectonics of the classic novel. It breaks with the canon and imposes another reception modality which provides obvious enjoyment to the reader. This work highlights all of the enunciative, linguistic and discursive phenomena that contribute to the authenticity of this work. Keywords: Stylistics, generic hybridity, intertextuality, enunciation scene, polyphony

\section{EXTENDED ABSTRACT}

Stylistics is a discipline that derives from the sciences of language. It aims to decrypt literary texts through a process that makes texts literary. To achieve its goal, this discipline makes use of concepts and methods borrowed from discursive linguistics, rhetoric, poetics, semiotics and many other areas of text science (Calas, 2015, p. 7). Stylistics is viewed as an eclectic field. Its field of inquiry relates to the literary fact as an enunciative act, a meaning-form which obeys a hermeneutic approach. It is therefore qualified to reveal the aesthetic character of all literary productions. However, this present stylistic study mainly focuses on poetics. It is a science of text which is contiguous to stylistics. Poetics aims to bring out the formal properties of literary creations. It is based on a set of approaches, among which, is intertextuality. In the stylistic analysis of literary texts, intertextuality is one of the most useful theories of poetics. This concept, which was coined by Julia Kristeva in the 1960s, is used in the studies of comparative literature, speech analysis, poetics and stylistics. It is defined as the relationship that one text has with another. As a discursive practice, intertextuality can be studied through various scales of verbal creation: through quotation, pastiche, parody, mise en abyme etc. These processes play an essential role in the African verbal creation. Indeed, since the brilliance of Ahmadou Kourouma's Les Soleils des independences and Yambo Ouologem's Le Devoir de violence, African romantic creation has continued to improve over the years. This constantly growing innovation, which translates into the textualization of the African sociolinguistic universe and other processes, contributes to an Africanization of the writing language. The recourse to the Ivorian origin contributes to the lexical connotation. Thus, the terms inserted by the authors in the writing language participate in the enrichment of the French language. One could say that relexification is at the heart of literary creation. It is materialized by the transposition of statements, by the insertion of oral ethno-texts in the novel. This language fabrication is a form of discursive subversion that results in an aesthetic of hybridity. The novel Le-fils-de-la-femme mâle 
is no exception to this standard. When we read it, it stands out that the author expresses his vision of the world through the incorporation of oral genres in the story. The typographical configuration shows that the enunciative foundations of the work rest on the structure of the traditional African tale. The marks of the tale are inserted in the discourse through processes such as italics and quotation marks. Despite the effort made by the romantic tale teller Maurice Bandaman to hide the tale's tracks, of the song and myth in the novel structure, the reader is able to perceive these oral intertexts through their characters. Through this process, the classic narration is interrupted. Furthermore, the insertion of the traditional tale into the structure of the novel is not based on any stable rule. Discontinuity leads the reading and the decoding of the message imparted by the novelist to the receiver. Viewed as a discourse, the work is based on an intertextual practice which overturns the architectonics of the classic novel. It breaks with the canon and imposes another reception modality that provides enjoyment to the reader. The dominance of formulas in the strata of this novel provides a basis for generic hybridity. The main purpose of this reflection is to highlight all of the enunciative, linguistic and discursive phenomena that participate in the renovation of this romantic work. Moreover, the examination of the utterance scene attests that the narration is supported by several vocal sources. It offers a hold on an exploitation of polyphony which goes beyond narration and results in the marks of reported discourse and autonymic modalization. The latter highlights the position of the enunciator with regards to his statement. In Ivorian novelist work, autonymic modalization is marked by quotation marks and italics. These linguistic signs underline the words that the author invented to construct his story. The lexical creativity undoubtedly gives originality to Maurice Bandaman's romantic discursiveness. This is certainly the reason why the mediation authorities awarded the author the "Grand prix littéraire de l'Afrique noire" when the book was published in 1993. 


\section{Introduction}

La création romanesque de Maurice Bandaman repose sur la textualisation du patrimoine linguistique akan. Cette opération, basée sur le matériel sonore, se solde par une œuvre qui épouse les canons esthétiques de l'oralité africaine. Une analyse des lois présidant à la fabrication de ce langage induit une analyse poétique. Ce travail qui s'inscrit dans la dynamique de la stylistique ne peut occulter la poétique. Discipline qui étudie le fait littéraire en tant que combinaison d'une forme et d'un sens, la poétique est l'expression de cette valeur qui éclaire l'art littéraire. Cependant, une réflexion sur la littérature impose la prise en compte de la littérarité. En partant de la forme vers la mise en forme, l'étude commande une démarche stylistique. Ces deux sciences contiguës visent le fonctionnement esthétique du texte. Tandis que la poétique s'intéresse au décryptage des formes littéraires pour en établir une typologie, la stylistique, quant à elle, étudie ces formes pour en proclamer la littérarité. Claire Stolz le confirme :

La poétique [...] cherche à percer les secrets de fabrication de la littérature, les secrets de la littérarité, c'est-à-dire de ce qui constitue un texte en œuvre littéraire. Cette recherche s'effectue tous azimuts depuis les aspects sociologiques jusqu'aux aspects purement langagiers qui relèvent de la stylistique. [...] La stylistique a donc des visées proches de la poétique. (2005, pp. 31-32)

La poétique et la stylistique coopèrent donc dans le champ des sciences du texte. À partir de cette synergie, l'étude explore la créativité langagière de Maurice Bandaman. L'art verbal de ce romancier-conteur repose sur des néologies et des cryptotypes responsables de certains faits de langue. Ils apparaissent comme le résultat de la pression exercée sur le signifiant. Le processus obéit à une indigénation du français. Une telle innovation langagière opère des bouleversements au niveau de la structure profonde du roman et révèle la part créatrice du romancier. Les transformations effectuées portent sur le genre, I'hypotexte et la thématique. Au-delà des subversions, celles-ci reposent sur la déviance, les variations et le style. Ces éléments déterminent la vision du monde et le génie du romancier. C'est pourquoi l'étude s'intéresse à l'usage que l'écrivain fait de la langue française. Elle explore les procédures langagières en jeu dans Le-fils-de-la-femme-mâle. Elle est structurée en trois principales parties. La première porte sur la poétique lexicale. Elle met l'accent sur la subversion linguistique. La deuxième 
étudie les modalités d'insertion des genres oraux dans le discours romanesque. La troisième s'intéresse à la polyphonie.

\section{La créativité lexicale, une caractéristique de la poétique de Maurice Bandaman}

Dire que la créativité lexicale est une marque de fabrique de l'œuvre romanesque de Maurice Bandaman, c'est démontrer que le romancier introduit dans la langue d'écriture de nouvelles unités lexico-sémantiques qui le connotent à la réception. Cette action qui obéit aux exigences de la connotent littéraire revitalise la créativité esthétique. Elle s'effectue à partir d'une violence sur l'écriture. Le romancier procède ainsi à une opération de déstructuration et de restructuration de la langue française. L'acte n'est pas sans conséquence sur l'architectonie du texte. Il se matérialise par l'insertion de néologies, c'est-à-dire l'entrée de nouvelles unités lexicales dans la langue. La linguistique distingue deux types de néologies qui sont la néologie de forme et la néologie de sens. Le phénomène se caractérise par le choix de certaines lexies pour traduire des réalités quotidiennes avec des voies nouvelles. Jean Dubois distingue les deux procédés par cette affirmation : «La néologie de forme consiste à fabriquer [...] de nouvelles unités ; la néologie de sens consiste à employer un signifiant de sens existant déjà dans la langue considérée en lui conférant un contenu qu'il n'avait jusqu'alors » (2012, p. 68). La néologie est un processus qui enrichit la langue d'accueil. Elle opère sur les structures du signifiant et du signifié. L'étude accorde le primat aux néologies de forme. Celles-ci se subdivisent en deux principales catégories qui sont la dérivation et la composition. Dans Le-fils-de-la-femme-mâle, la seconde forme donne lieu à des lexies qui authentifient l'art verbal du romancier. Elles se signalent dans le dispositif énonciatif par des connotations topolectales révélatrices du contexte socioculturel dans lequel évoluent les actants. Mais avant de décrire ce phénomène dans l'œuvre, il est nécessaire de le définir. La composition désigne un procédé de création lexicale mis en œuvre par la juxtaposition de divers morphèmes lexicaux ou grammaticaux. Les morphèmes ainsi juxtaposés sont susceptibles d'avoir, par eux-mêmes, une autonomie dans la langue. Techniquement, la composition se traduit par des termes dont les composantes sémantiques sont complémentaires et reliées au niveau de la graphie par un trait d'union ou une préposition. Chez Maurice Bandaman, le phénomène s'observe dans le système de la personnalisation. Ce système porte sur l'analyse des actants dans la fiction littéraire. Le procédé grammatical est indissociable de l'énonciation. II inclut l'onomastique (les noms propres et les appellatifs), les déterminants possessifs et les 
pronoms personnels. Chez l'auteur ivoirien, la plupart des appellatifs sont constitués analogiquement à partir des synthèmes provenant de sa langue maternelle, comme en témoignent les occurrences suivantes :

Beau-chasseur-du-pays-des-vivants ${ }^{1}$, viens par ici. (Bandaman, 1993, p. 12) La danse des hommes-pour-qui-la-mort-est-un-frère. (Bandaman, 1993, p. 55)

Les enseignements de Maître-Alua-le-Chien ou l'apprentissage de l'Amour et de la Fidélité. (Bandaman, 1993, p. 75)

Les enseignements de Maître-Bwalodohé-le-Bélier ou l'apprentissage de la force et de la tolérance. (Bandaman, 1993, p. 81)

Les enseignements de maître-Kotokoly-la-pie ou l'apprentissage de la poésie. (Bandaman, 1993, p. 97)

L'onomastique repose sur une valeur sémantico-référentielle. Elle est renforcée par la disposition morphosyntaxique des lexies. Les appellatifs et les noms sont composés de mots reliés par des traits d'union. Ils visent à agglutiner les termes occurrents. Dans I'univers culturel baoulé, ces expressions ont valeur de qualification. Elles réfèrent à des réalités qu'on ne peut désigner par les mots dérivés de la langue de Molière.

Ce processus de désarticulation du français est rehaussé par des africanismes lexématiques. En marge des néologies de forme, le langage du romancier est enrichi par de nombreux emprunts. Ils sont fondés sur une matrice extérieure à la langue d'écriture. Par définition, l'emprunt linguistique est l'incrustation de certains items d'une langue à une autre. Ces composants s'enracinent dans la structure lexicale, phonétique et grammaticale de la langue d'accueil. Ils participent à la communication dans un contexte unilingue ou bilingue. Dans cette œuvre, les emprunts d'origine akan sont les dominantes. Ils appartiennent au répertoire baoulé, groupe ethnique du romancier. Le recours à ces emprunts souligne la volonté de l'auteur de réinventer, à partir du français, un langage spécifique, de promouvoir sa langue maternelle. C'est le cas des exemples suivants :

- J'ai prié mon Bohoussou (génie de la chasse) ce matin ; c'est sûr que j'abattrai une biche cette nuit. (Bandaman, 1993, p.11)

Ehé ! Gnamien Kpli! (Grand Dieu) vraiment des choses étranges et pourtant

1 Les soulignements mettent en relief les phénomènes linguistiques analysés dans le corpus d'étude. 
varies existent au monde. (Bandaman, 1993, p.13)

- O merci, Nanan (Grand père). (Bandaman, 1993, p. 20)

Les hommes ne voulurent pas mécontenter le «ummien» (l'âme) du défunt en l'obligeant à quitter le village. (Bandaman, 1993, p. 38)

Vois-tu, là-bas, derrière cette colline, les cercueils qui traînent ? Eh bien, tous appartiennent aux jeunes et aux féwas qui n'ont pas le droit de traverser ce fleuve. Vois-tu, tu n'es pas le seul à être refoulé de Blôlo. (Bandaman, 1993, p. 46)

Stylistiquement, l'incorporation des lexies dialectales dans le discours romanesque crée des connotations qui en font un label. En vérité, le lecteur a affaire à des pérégrinismes. La savante insertion de ces pérégrinismes dans les nervures du texte démontre le génie du créateur. En linguistique, le pérégrinisme désigne une forme lexicale dérivée d'une langue et employée dans une autre sans assimilation. Ils expriment des réalités propres à la culture d'origine et considérées comme inexprimable dans la langue réceptrice. Ce savoir-faire, dit Gérard Noumssi (2009, p. 43), s'inscrit dans le process d'emprunt de compétence. Il exemplifie la détermination de l'auteur à transposer des relia culturelles et des expressions que le lexique français ne saurait exprimer.

En outre, on observe deux formes de diglossies. II s'agit, d'une part, des diglossies textuelles et, d'autre part, des diglossies littéraires. La première est le processus par lequel l'auteur fait jouer habilement deux langues sur une aire textuelle. C'est au niveau scriptural que l'on apprécie cette technique créatrice. Sur la même surface de texte, le romancier emploie concurremment deux langues. Par ce procédé, il travaille la langue du colonisateur de l'intérieur. La tension entre les deux langues génère des statalismes et des calques de tous ordres, ainsi que l'attestent les énoncés suivants :

-Tooo to lia lia too

Tooo to lia lia too

Awé blé atomoliééééé

Tooo to lia lia too

N’zué wa yi wa héé

Bla man yé non!

O mère Buffle à la beauté parfaite

L'eau a, ici, envahi la terre

Viens qu'on la boive! (Bandaman, 1993, p. 83) 
La conjonction des langues dans le récit est le signe caractéristique de l'hybridité langagière. Les diglossies textuelles sont soutenues par les diglossies littéraires. Elles se concrétisent par une traduction littérale des mots et des expressions du baoulé dans la langue française. Ces marques linguistiques cristallisent le système figuré dans la création verabale et ne sont pas des épiphénomènes littéraires. Elles découlent de la fixation des langues maternelles dans des langues d'écriture étrangère. Dans ce type de création, l'hypertexte devient un « palimpseste » qui porte les marques d'une première langue. Genette entend par là « toute relation unissant un texte $B$ [...] à un texte antérieur $A[. .$.$] sur lequel il se greffe d'une manière qui n'est celle du commentaire$ » (Genette, 1982, p.12). Bien que les textes soient composés en langue française, ils portent le sceau des langues maternelles et véhiculent des réalités extra-occidentales. Cette hétérogénéité peut brouiller la communication et perturber la réception. De ce qui précède, il résulte que l'œuvre est ancrée dans la géographie culturelle ivoirienne. Son originalité est exprimée par le fait qu'elle charrie le lexique akan.

\section{Analyse stylistique des marques du palimpseste dans Le-fils- de-la femme-mâle : un conte-romanesque}

L'analyse stylistique du palimpseste explore la manière par laquelle le discours littéraire combine différents types et genres de discours par la technique de la cooccurrence et de la transformation. De toutes les productions artistiques, seuls les textes littéraires ont la capacité d'intégrer en leur sein les autres formes d'art. Tiphaine Samoyault, dans la conclusion de son ouvrage sur l'intertextualité, énonce les pistes d'une analyse stylistique de l'intertextualité en mettant un accent sur la circularité des intertextes :

Pour l'analyse stylistique des textes, l'intertextualité comme méthode peut être convoquée pour le repérage de disjonctions ou d'éléments hétérogènes. [...] Une stylistique de l'intertextualité semble également productive : en faisant l'inventaire des occurrences intertextuelles pour préciser leurs indices contextuels et leurs configurations formelles, il devient possible d'envisager les liens de la littérature avec elle-même comme des opérations techniques particulières. (Samoyault, 2013, p. 112)

La stylistique de l'intertextualité vise à montrer la manière dont les œuvres littéraires intègrent des discours composites. Chez Maurice Bandaman, ces relations sont nouées 
sous la forme d'un collage littéraire. Ce procédé de création relève des arts plastiques. En pratique, le collage est l'incorporation de différents motifs de papier pour créer une toile. Ces éléments hétéroclites s'ingénient pour constituer une réalité hybride. Dans l'art littéraire, il se matérialise par l'insertion d'intertextes cosmopolites. Les fragments sont intégrés sous la forme de citations, d'emprunts déguisés, de pastiche, etc. Le-filsde-la-femme-mâle mobilise plusieurs intertextes oraux dont le conte, les proverbes, le chant, la poésie etc. Ce brassage en fait un creuset transgénérique. L'idée est mise en évidence par cette formule inaugurale :

Ecoutez!

Ecoutez!

Gens d'ici

Et gens d'ailleurs!

Ecoutez ma voix!

Je vais vous dire une histoire

Cette histoire est un conte

Cette histoire est comme un conte !

[...]

Le vrai et le faux sont un couple !

Gens d'ici

Gens d'ailleurs!

Ecoutez ma voix!

Il était une fois... (Bandaman, 1993, p. 6)

Les énoncés par lesquels s'ouvre le roman sont des formules spécifiques au conte africain. De nombreuses occurrences témoignent de la matérialité de cet art. Elles sont inscrites par des expressions telles que : «écoutez », « gens d'ici », " gens d'ailleurs », " écoutez ma voix ». Ces formulaïques permettent au conteur de capter l'attention de l'auditoire. Elles sont soutenues par les énoncés « Je vais vous dire une histoire», « cette histoire est un conte ». Ces énoncés apparaissent comme des formules instituées. Ils sont assortis de délocutés « il », « elle » observables dans les phrases : « elle dit vrai », " elle dit faux», « Il était une fois... ». Ces délocutifs soulignent le caractère impersonnel et atemporel du conte. Le langage ainsi produit fait office d'une modalisation zéro. En plus, dans cet extrait, certaines occurrences attestent que le discours littéraire échappe au critère de la vériconditionnalité. Elles sont mises en évidence par les constructions : «Le vrai n'est pas forcément vrai », «Et le faux n'est pas forcément faux », « Le vrai et 
le faux sont un couple ». D'un point de vue pragmatique, ces énoncés montrent que le discours produit par le romancier ne s'inscrit ni dans la catégorie du vrai ni dans la catégorie du faux. II relève simplement de la fiction. Réfléchissant sur le caractère véridique ou mensonger des énoncés dans les genres de discours, Jean-Michel Adam affirme que l'évaluation se fait selon deux régimes:

L'évaluation de la valeur de vérité des énoncés [se fait] selon deux régimes pragmatiques : celui de la vériconditionnalité qui repose sur l'opposition du vrai et du faux /mensonger et celui de la fictionnalité, qui apparaît comme un régime du ni vrai ni faux dans lequel on peut intégrer la métaphoricité. (Adam, 2008, p. 70)

La création littéraire est attachée à un genre de discours. Elle est soutenue par les figures de style. Ces éléments propres à l'institution littéraire échappent aux critères de la vériconditionnalité. Ils sont perceptibles dans le roman à travers les énoncés :

Approchez vous-mêmes vos yeux et fixez bien cette plaie : des squames mycosiques superposées et super-perforées par des milliers d'entozoaires ; un lac de pus où grouillent des grenouilles; des têtards bien musclés ; un lac où pullulent des asticots gros comme des têtes d'enfants; des fourmis dont la tête atteint la taille du gland d'un pénis adulte ; des serpents longs comme la distance de Pretoria à Washington via Paris; des moustiques aux ailes comme des rémiges d'aigle; des têtards cornus comme un buffle mandingue. (Bandaman, 1993, pp. 13-14)

Ces énoncés allient figures microstructurales et macrostructurales. La métaphore et la comparaison sont les figures microstructurales dominantes. Pour Molinié, « elles se signalent de soi, elles sont obligatoires pour l'acceptabilité sémantique et isolables sur des éléments formels déterminés et fixes » (Molinié, 2005, p. 96). La première se signale à travers le syntagme nominal « un lac de pus », puis dans l'énoncé verbal : "des fourmis dont la tête atteint la taille du gland d'un pénis adulte». Cette métaphore fait abstraction du comparant. Elle n'affecte pas le comparé mais agit sur certains prédicats. Alors que dans la première métaphore, le comparé et le comparant sont tous matérialisés. La métaphore est l'un des tropes les plus représentatifs du discours littéraire. À la métaphore, il faut associer la comparaison. Elle est introduite par l'adverbe de manière « comme » itéré six fois dans l'exemple. Les figures microstructurales qui 
affleurent à la surface de l'œuvre sont le prélude d'un discours hyperbolique. L'hyperbole est une figure qui se caractérise par le grossissement des faits. Brigitte Buffard-Moret la définit comme " une figure d'amplification, qui consiste en une exagération de l'expression destinée à produire une forte expression » (2015, p. 97). Dans cet extrait, le phénomène enveloppe tout le discours. Chaque énoncé est assorti d'un vocabulaire intensif doublement amplifié. L'énoncé nominal : « des serpents longs comme la distance de Pretoria à Washington via Paris » en un exemple. La permanence de cette figure oblige le lecteur obvie à convertir constamment ces énoncés «à un degré plus bas de désignation » (Molinié, 1992, p. 166). Aussi la prégnance des figures d'amplification dans le roman est-elle une marque de littérarité. Elle est typique à la scène d'énonciation du merveilleux et du surnaturel. C'est pourquoi l'hyperbole plonge le lecteur dans un monde où dominent les forces surnaturelles. Dans ce cas de figure, la scénographie n'amène pas le destinataire « à hésiter entre une explication naturelle et une explication surnaturelle [...] les récits merveilleux sont des récits où le surnaturel est accepté comme tel à côté du naturel. II n'y a pas de rupture entre les deux » (Todorov, 1970, p. 37). Nous en voulons pour preuve le voyage effectué par Awilimba dans le « ventre de la terre ». Ainsi que le témoigne le narrateur:

Une grande lumière fendit la nuit et la terre ouvrit son ventre. C'est donc dans le ventre de la terre qu'Awilimba marchait, son fusil sur l'épaule, inconscient du changement d'univers. Le ventre de la terre était large, profond, spiralé, stratifié. Awilimba traversa une première strate de sept niveaux [...]. Puis vint une deuxième strate également de sept niveaux [...]. Enfin une troisième strate de sept autres niveaux aux couleurs arcen-ciel. (Bandaman, 1993, pp. 11-12)

La description que fait le narrateur repose sur le merveilleux. Le grossissement textuel est mis en œuvre au moyen des métaphores verbale, prépositionnelle et attributive. Elles sont perceptibles dans les énoncés verbaux : « Une grande lumière fendit la nuit et la terre ouvrit son ventre », « Le ventre de la terre était large, profond, spiralé, stratifié ». La topographie de cet univers est propre au conte. Le sujet porte sur des actions extraordinaires réalisées par un héros surhumain. À travers cette topographie, le romancier transporte le lecteur dans un monde où dominent les formes superlatives tant sémantiques que syntaxiques. La plupart des personnages évoqués dans l'œuvre sont dotés d'une force, de traits distinctifs unique et hors du commun. L'intervention du fantastique unit le monde des humains à celui des forces surnaturelles et à celui 
des dieux. Ces marques textuelles reposent sur des présupposés qui répondent aux attentes des adeptes du conte traditionnel africain. Celui-ci est attaché à un dispositif énonciatif bien installé dans les savoirs partagés. L'intégration du conte dans le roman est une innovation. Cependant, ce genre de discours n'est pas la seule valeur distinctive, le roman est ponctué de chants et de parémies. Le chant est une pièce versifiée, caractérisée par des stances et reliée par un refrain. Chez Maurice Bandaman, le chant et la poésie présentent la même configuration. Par ailleurs, ces chants rituels sont exécutés à l'occasion des cérémonies mortuaires, ainsi que le montrent ces extraits :

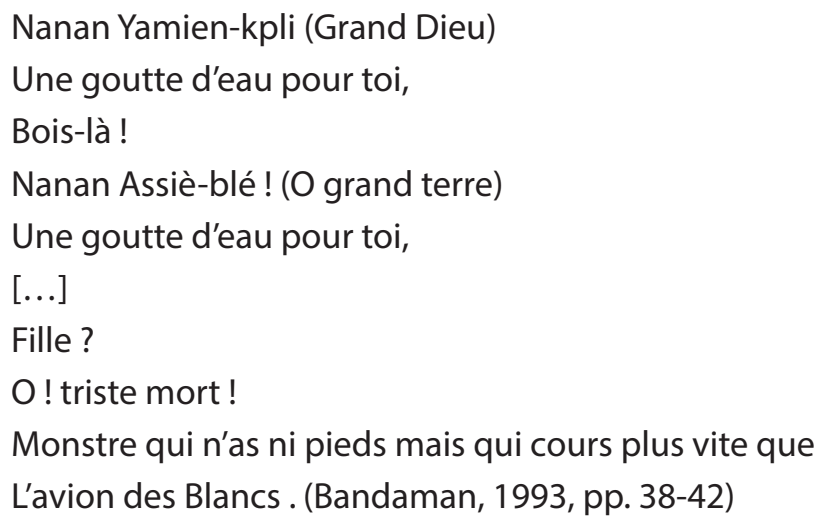

Au plan architectural, le poème rituel se caractérise par une organisation débridée. Le poème est constitué de monorème, de phrase dirème, de phrase nominale et de phrase complexe. Elles sont revêtues par le baoulé. Les lexies « Nanan Yamien-Kpli » et « Nanan Assiè-blé » le justifient. À travers la poésie rituelle, « le poète au verbe de feu » invoque les mânes des ancêtres dans ce qui s'apparente à une oraison funèbre. À cette poésie, il faut ajouter celle de Maître-Kotokoly-la-Pie. Quant aux parémies, on les relève dans les énoncés suivants :

Nos grands-parents disaient: Quand un lépreux se décide à livrer une bataille contre un bien-portant, convaincs-toi qu'il a la main sur une pierre. (Bandaman, 1993, p. 58)

Qui voyage à dos d'éléphant ne doit pas craindre la rosée. (Bandaman, 1993, p.160)

Par leur mode d'énonciation, les énoncés proverbiaux sont anonymes. Les proverbes sont des éléments géoculturels qui déterminent la fonction sociale du langage. Ils sont 
repérables, quel que soit le travail effectué pour les dissimuler. La récurrence du chant, du poème et des parémies dans le roman est conforme aux normes de l'institution littéraire africaine. Cette institution admet un mode d'énonciation hybride. Le constat se justifie par le fait que l'insertion des proverbes, de l'épopée, du mythe, des chants etc. dans le conte est un procédé spécifique à la littérature orale africaine. J-M. Adiaffi le souligne en ces termes :

La tradition orale africaine ignore absolument la notion de genre. Les contes sont accompagnés par la musique, celui qui récite le conte joue de la comédie. II est fréquent d'arrêter le conte par des charades, des proverbes, par tout un ensemble de jeu qui permettent au conteur de reprendre son souffle. II n'y a pas de genre. L'épopée, le conte, la fable, la devinette [...] tout est mélangé. ${ }^{2}$

Maurice Bandaman a imité la tradition orale pour créer un roman informe et original. L'hypotexte montre que l'art oral africain est un terreau pour la littérature contemporaine. De nos jours, les romans africains sont constamment interrompus par des poèmes, de chansons rituelles souvent accompagnées de danse. Le romancier donne vie à cette réalité à travers l'exécution de la danse des membres de la société secrète. «Afonssou », «Adjanou » et leurs compagnons l'illustrent:

\section{Zoua! \\ Zoua! \\ Zoua! \\ Zoua-Zoua! \\ Nous dansons la danse des morts... (Bandaman, 1993, p. 55)}

Cet extrait constitué de monorèmes laissent entrevoir un discours onomatopéique. La danse scelle le pacte entre les membres de la confrérie et leurs aïeux. Ils renouvellent leur serment à la faveur de cette cérémonie. Ils préfèrent mourir plutôt que de profaner le secret que leur ont légué ceux-ci. La création romanesque de Maurice Bandaman est une pratique intertextuelle qui rompt avec la lecture linéaire. Sur ce point, Anne Claire Gignoux affirme : « la linéarisation est une évidence importante pour comprendre les mécanismes de la lecture intertextuelle. Le texte inséré vient rompre le fil du texte

2 Jean-Marie Adiaffi, Extrait de l'entretien avec Bernard Magnier à Paris, en Novembre 1982 dans le cadre du Grand Prix littéraire d'Afrique noire. 
final, qu'il apparaisse en italique ou intégré dans ce dernier » (2005, p. 39). Le roman oscille entre le mythe, la légende et l'épopée. Ces intertextes sont décelables par l'intervention des personnages tels que « Mamie-Watta » l'une des mères d'Awilimba 3 et «Bla Yassoua » l'instigatrice de la marche des femmes pour la libération de leurs époux emprisonnés sur une île par le dictateur « Nanan Aganimo ». Il existe dans cette séquence de l'œuvre des faits qui rappellent la légende baoulé. À l'instar d'Abla Pokou qui a sacrifié son fils unique pour sauver son peuple de l'agresseur, «Bla Yassoua », femme charismatique, accepte de calmer la colère de l'océan et assouvir le désir de " Mamie-Watta » la reine des eaux en sacrifiant son fils unique. Il existe également une corrélation entre la marche des femmes sur la prison de Grand Bassam et celle initiée par Bla Yassoua. Ces références montrent que le roman se nourrit de l'épopée. C'est un genre oral qui s'inspire du réel mais avec un grandissement des faits. Pour rester dans la logique du conte, le romancier clôture son roman par une formule consacrée :

\section{EEEEEhhhhhhh! Gens d'ici}

Et gens d'ailleurs !

Vous avez écouté mon conte

[...]

Bonne nuit à chacun

Aux enfants des rêves fantastiques

Aux adultes grandes méditations... (Bandaman, 1993, p. 169)

Ces énoncés annoncent la fin du conte. Le conteur invite les personnes adultes à la méditation et souhaite une douce nuit aux enfants. Cette formule est propre aux contes africains. Les syntagmes nominaux «Bonne nuit à chacun », « Aux enfants des rêves fantastiques » et "Aux adultes grandes médiations » attestent la participation de la communauté aux séances de contes. La conjonction des genres oraux dans le roman fait de la créativité langagière de Maurice un discours polygénérique. Elle est conforme à la poétique de la tradition orale africaine qui est par essence « un genre sans genre ».

\section{Discours rapporté et modalisation autonymique : une mise en scène polyphonique dans l'art discursif du romancier-conteur}

L'originalité de l'oeuvre romanesque ne s'observent pas seulement dans la créativité lexicale, elle réside également dans le traitement des voix. Il existe dans l'écriture de 
Maurice Bandaman des éléments qui laissent entrevoir une empreinte polyphonique. Ce phénomène qui s'observe à deux échelles est propre au genre de discours et à la visée illocutoire du romancier-conteur. La première est indissociable du mode narratif. Elle se caractérise par la coprésence de deux instances narratives et par l'intervention de plusieurs personnages. Comme la plupart des romans, Le-fils-de-la-femme-mâle a préservé la narration traditionnelle à travers l'emploi du délocutif « il ». Cette manière de raconter est à l'actif du narrateur extradiégétique. Extérieur à l'histoire, il se distingue par son omniscience et son caractère impersonnel. À côté de cette instance, il convient de souligner la présence du narrateur homodiégétique qui s'illustre par le pronom personnel « Je ». Le romancier, par cette action, transpose la scénographie du conte traditionnel dans la structure du roman. Ce travail infléchit les catégories de la narration classique et offre une assise à la polyphonie littéraire. Cela se justifie par le fait que ces occurrences se disputent le monopole de la narration. Pour étayer cette assertion, citons quelques lignes de Pierre N'da :

Le romancier ivoirien [...] a conservé le narrateur extradiégétique, omniscient et impersonnel « il » courant dans les romans; mais il a ajouté un autre narrateur, un narrateur homodiégétique qui dit « je » et qui livre une narration à la manière du conteur africain ou de griots. Il y a donc au moins deux narrateurs explicites dans ces récits et il est loisible de parler de narration polyphonique. (N'da, 2003, p. 52)

Le style de la seconde instance est identique à celui des aèdes africains. Il calque le roman sur l'institution discursive africaine. L'œuvre conjugue donc les outils discursifs propres au roman et à l'art verbal africain. À titre d'exemple, la scène d'énonciation du conte se perçoit par la multiplicité des énonciateurs. Par moment, des personnages tels que " Afonsou », « Nanan Yablé », « Mami Watta », « Awilimba Tankan » n’hésitent pas à s'approprier la parole pour raconter certains événements. Ils se substituent au narrateur principal et deviennent eux-mêmes des narrateurs transparents. Le principe est conforme au rituel qui préside à l'énonciation du conte africain. Car, il y a des moments où le récit est interrompu par l'intrusion de certains auditeurs qui deviennent eux-aussi des énonciateurs. Cette polyphonie liée à la scène d'énonciation du conte concerne la première échelle. Mais, le traitement de la polyphonie ne se limite pas à ce palier. D'autres indices polyphoniques sont détectables à travers le discours rapporté et la modalisation autonymique. 
Le premier peut se définir comme l'intégration d'une énonciation dans une autre énonciation. Typologiquement, elle se traduit par l'inscription du discours cité dans le discours citant. La tradition grammaticale distingue trois types de discours rapporté. Le style direct, le style indirect et le style indirect libre. Chez le romancier ivoirien, les deux premiers sont significatifs. On le remarque dans ces exemples :

Mais Awilimba comprit qu'une telle fantaisie ferait la honte de ses parents qui s'entendraient dire : « Sorcier ! Vous avez non seulement mangé votre enfant, mais vous avez encore dévoré sa dépouille. II n’a pas de sépulture, la preuve ! » (Bandaman, 1993, p. 44)

Le discours direct se démarque des autres formes par sa typographie. Il est marqué par la mise en scène de deux situations d'énonciation. Ici, le narrateur extradiégétique est le rapporteur de l'énonciation « Mais Awilimba comprit qu'une telle fantaisie ferait la honte de ses parents qui s'entendraient dire : ». Par la suite, il délègue la responsabilité au locuteur du discours direct. Dans l'énoncé, le deux points occupe une fonction démarcative. Il précède les guillemets qui constituent la frontière entre les deux domaines énonciatifs et annoncent le passage au discours direct. Le discours cité est placé en position de complément d'objet du verbe de parole « dire ». Le style direct est un simulacre verbo-textuel par lequel la parole citée conserve sa propre actualisation. Elle peut être constituée d'énoncés de diverses natures : actes de langage, interjection, phrases incomplètes, langues étrangères, etc. Pour l'interpréter, il faut se référer au contexte d'énonciation. Quant à la parole citante, elle renseigne sur les personnages, la chronographie, la topographie etc. Le style direct fonctionne comme une greffe intertextuelle circonscriptible par le lecteur. L'opérationnalisation du discours direct laisse entrevoir deux domaines énonciatifs autonomes. En marge du style direct, le style indirect, caractéristique de l'énonciation romanesque, renferme une polyphonie.

- Hommes, femmes et enfants!Tendez vos oreilles et écoutez-moi. Notre chef me charge de vous dire qu'Akandan, le mari d'Assouman, le fils de Nanan N'san Akopolé a quelques paroles dans son ventre. Et ces paroles enroulées dans son ventre sont des accusations! (Bandaman, 1993, p. 58)

- Ehé ! s'exclama N'san, le héraut. Akandan dit que ce n'est pas tout le village qu'il a vu ou entendu voler, violer, tuer ou commettre un adultère, mais quelques individus. (Bandaman, 1993, p. 58) 
Ces extraits montrent que les deux énonciations intègrent le discours cité dans le discours citant. L'énoncé cité n'a plus d'autonomie. Dans la narration, il n'y a plus que le je-origine. Quant au repère temporel, il est celui du discours rapportant. L'énoncé rapporté apparaît comme une complétive d'objet direct. Les deux énoncés sont introduits par le verbe de parole «dire ». Seul le sens de ce verbe permet d'interpréter la proposition comme une citation. Il est en général suivi de la conjonction «que ». Les exemples suivants l'attestent : « Notre chef me charge de vous dire qu'Akandan, le mari d'Assouman... », «Akandan dit que ce n'est pas... » Le discours indirect est une opération de paraphrase. Il laisse entendre deux différentes sources énonciatives. Mais la polyphonie n'est pas que dans le discours rapporté. Elle se présente de différentes manières dans les énoncés. L'un des avatars de la polyphonie est la modalisation autonymique. Elle consolide la polyvocalité dans la créativité romanesque.

La modalisation autonymique est l'attitude adoptée par l'énonciateur à l'égard de son énoncé. Chez Maurice Bandaman, la modalisation est réparable par l'italique et les guillemets. Dans Le-fils-de-la-femme-mâle, l'on observe un emploi récurrent, voire ennuyeux de ces marques typographiques. Elles occupent une place importante dans la communication que l'énonciateur établit avec le lecteur obvie. Il en va ainsi des occurrences suivantes :

Tu iras au Ghana avec tes « kentes » et tes livres; ils te seront d'une grande utilité là-bas. (Bandaman, 1993, p. 46)

" Attô » (ce n'est pas vrai), mon fils a été empoisonné ; je sais par qui et pourquoi. J'ai donc eu raison de faire partir ma bru dans son village. (Bandaman, 1993, p. 49)

Le mien porte depuis sa naissance trente-trois dents et parle comme si un « vieillard était assis dans son ventre ». (Bandaman, 1993, p. 61)

On fit descendre la bière dans la tombe et « pou ! pou ! pou ! » on la boucha. [...] On posa quelques objets ayant servi à l'usage du disparu puis « tohou ! tohou ! tohou ! » trois bons coups de fusil furent tirés dans l'air pour chasser le mort du monde des vivants (Bandaman, 1993, p. 44). 
Le romancier ivoirien a recours aux guillemets à des fins diverses. Les extraits des pages 46, 49 et 61 montrent qu'il marque une certaine distance à l'égard des termes employés. En effet, les lexies « Kentes », « Attô » sont empruntées au vocabulaire baoulé. En les mettant entre guillemets, il les présente comme inappropriées par rapport à la langue d'écriture. D'ailleurs, l'explication des mots est mise entre les parenthèses. Ces dernières sont aussi des instruments de l'autonymie. Les guillemets, quant à eux, soulignent les disjonctions syntaxiques. Comme l'indique l'exemple de la page 61 « un vieillard était assis dans son ventre ». Cet énoncé recèle un contenu asymétrique qui s'oppose au langage ordinaire. Dans les autres cas, la modalisation autonymique par les guillemets est mise en évidence par des expressions onomatopéiques. Les lexies « pou ! pou ! pou !», « tohou ! tohou ! tohou !» ont été créés par le romancier pour imiter le bruit du marteau et des coups de fusil. La transposition des structures du baoulé dans la syntaxe de la langue française concourt à une esthétique de la déconstruction du langage. Celle-ci est en partie liée au triomphe du langage onomatopéique, I'une des particularités des langues vernaculaires. Le romancier n'hésite pas à utiliser l'italique. Selon Dominique Maingueneau : «Comme les guillemets, l'italique s'emploie aujourd'hui à la fois pour l'autonymie et pour la modalisation autonymique. [...] Rien n'empêche donc de cumuler guillemets et italique. Mais ce cumul est plutôt réservé à la presse écrite qu'à la littérature » (2015, p. 174). C'est un marqueur de modalisation autonymique.

L'italique est incorporé dans l'énoncé pour marquer le changement de caractères, lequel changement peut être connotatif. L'œuvre se singularise par deux emplois distincts de l'italique. Le premier résulte de la pratique intertextuelle et le second de I'individuation. Puisque, dans l'art oratoire du romancier, l'italique sert de pont entre les différents genres de discours incorporé. La transcription en italique des paroles « du poète au verbe de feu Nanan Yablé » marque la frontière entre le récit et la poésie. Il en est de même des stances produites par Maître-Kokoti-le-porc. Elles mettent en évidence la place qu'occupe la chanson dans l'œuvre. La traçabilité du conte dans l'œuvre est rendue possible par l'italique qui permet de le distinguer. Le second découle d'un usage individué en ce que le romancier cumule les deux marques typographiques.

\section{O frère Assamoi !}

Pour toi nous dansons la danse des morts

[...]

La mort si nous étions contraints de le faire pour ne pas 


\section{Profaner leur secret}

Zoua!

Zoua!

Zoua!

Zoua-zoua!

Nous dansons la danse des morts... (Bandaman, 1993, p. 55)

Si dans le premier cas, l'italique permet de distinguer les genres insérés dans le récit, il convient de souligner que dans le dernier extrait, l'auteur conjugue l'italique et les guillemets. Cet usage est une forme de transgression. Car dans un texte, l'italique est réservé pour les lexies étrangères. II permet de séparer une unité linguistique sur le fond du texte. En ce qui concerne les guillemets, ils sont utilisés par l'auteur pour exprimer un avis réservé quant à l'emploi d'un terme. Même si rien n'empêche le recours simultané à l'italique et aux guillemets, il convient de noter que cet usage est réservé à la presse écrite. La cumulation des deux marqueurs dénote de la capacité du discours littéraire à absorber les autres types de discours.

\section{Conclusion}

En guise de conclusion, retenons que l'œuvre romanesque de Maurice Bandaman se distingue par l'utilisation de matériau lexical. Le romancier exploite les ressources de la langue de sorte à créer un langage qui lui est propre. L'introduction des néologies et des africanismes lexématiques confère au discours produit une épaisseur socioculturelle dont les relents soulignent son ancrage dans l'oralité africaine. Cette action sur la langue démontre le savoir-faire du créateur. II plie la langue d'écriture à ses exigences. Mais au-delà, le romancier montre qu'il maîtrise les codes en usage dans les deux langues.

Le roman est le résultat d'une pratique intertextuelle. II mobilise plusieurs genres et types de discours. En s'inspirant des ressources de la culture baoulé, il innove par une écriture à cheval sur la tradition orale et sur le roman classique. Par rapport aux canons scripturaux du roman classique, Le-fils-de-la-femme-mâle se signale par son caractère transgressif et subversif. Du fait de l'incorporation des genres oraux, le langage apparaît fragmenté. il rompt avec l'horizon d'attente et impose des modalités axées sur la discontinuité. 
Évaluation : Évaluation anonyme par des pairs extérieurs.

Conflit d'intérêts : L'auteur n'a aucun conflit d'intérêts à déclarer.

Subvention : L'auteur n'a reçu aucun soutien financier pour ce travail.

Peer-review: Externally peer-reviewed.

Conflict of Interest: The author has no conflict of interest to declare.

Grant Support: The author declared that this study has received no financial support.

\section{Bibliographie}

Adam, J-M. (2008). La Linguistique textuelle. Introduction à l'analyse textuelle des discours, Paris : Armand Colin.

Bandaman, M. (1993). Le-fils-de-la-femme-mâle, Paris : L'Harmattan.

Buffard-Moret, B. (2015). Introduction à la stylistique, Paris : Armand Colin.

Calas, F. (2015). Leçons de stylistique, Paris : Armand Colin.

Dubois, J. et al. (2012). Le Dictionnaire de linguistique et des sciences du langage, Paris : Larousse.

Genette, G. (1982). Palimpsestes. La littérature au second degré, Paris : Seuil.

Gignoux, A. C. (2005). Initiation à l'intertextualité, Paris : Ellipses.

Maingueneau, D. (2015). Manuel de linguistique pour les textes littéraires, Paris: Armand Colin.

Molinié, G. (1992). Dictionnaire de rhétorique, Paris : Livre de poche.

Molinié, G. (2005). Éléments de stylistique française, Paris : PUF.

N'da, P. (2003). L'Écriture romanesque de Maurice Bandaman ou la quête d'une esthétique africaine moderne, Paris :

L'Harmattan.

Noumssi, G. M. (2009). La Créativité langagière dans la prose romanesque d'Ahmadou Kourouma, Paris : L'Harmattan.

Samoyault, T. (2013). L'intertextualité. Mémoire de la littérature, Paris : Armand Colin.

Stolz, C. (2005). Initiation à la stylistique, Paris : Ellipses.

Todorov, T. (1970). Introduction à la littérature fantastique, Paris : Seuil. 